\title{
Rabies Prevention in Asia: Institutionalizing Implementation Capacities
}

\author{
Mary Elizabeth G. Miranda and Noel Lee J. Miranda
}

\begin{abstract}
Rabies in Asia and Africa contributes to over $99 \%$ of human rabies deaths that occur in the world today. The vast majority or $60 \%$ of these deaths are in Asia. Practically, more than four billion people in Asia or about $60 \%$ of the world's population are at risk of getting rabies where an estimated $96 \%$ of documented human cases are from an infected dog bite. Canine-mediated rabies is one of the few communicable diseases that can possibly be eliminated by currently available vaccines and tools for veterinary and public health interventions. With a more comprehensive and integrated approach, it is expected that dog rabies will be eliminated in target areas, and there will be an eventual decline and disappearance of human rabies cases. The burden of rabies is primarily on human health but the disease control has to be focused on the animal source. The ultimate goal of a truly regional disease program is to control and eliminate dog-mediated rabies and protect and maintain rabies-free areas in Asia. Current regional efforts aim to strengthen the intercountry coordination, and technical and institutional capacities to manage dog rabies elimination programs. The regional and national implementation efforts provide strategic direction and cooperation to ensure successful implementation of rabies control measures and eventual elimination. The focus areas include human rabies prevention through pre- and postexposure prophylaxis, mass dog vaccination, surveillance and epidemiology, laboratory diagnostic capability, public awareness and risk communication, legislation, dog population management, and establishment and protection of rabies-free zones/
\end{abstract}

M. E. G. Miranda ( $\triangle)$

Research Institute for Tropical Medicine, Muntinlupa City, Philippines

N. L. J. Miranda

Research Institute for Tropical Medicine, Muntinlupa City, Philippines

World Veterinary Association Zoonotic Diseases Working Group, Manila, Philippines 
areas. Existing mechanisms for implementation, when applied, give emphasis on One Health collaborations.

\section{Situation in Asia and Regional Initiatives}

For most countries in Asia, canine rabies is endemic and the majority of human rabies exposure results from dog bites particularly among children. The estimated number of human deaths across Asia and Africa is approximately 59,000, with over 3.7 million disability-adjusted life years (DALYs) and 8.6 billion USD economic losses annually [1]. The vast majority of these deaths is in Asia (59.6\%). India, with $35 \%$ of human rabies deaths, accounted for more deaths than any other country. Rabies is often neglected when health and agriculture (animal health) agenda and budgets are set even if the costs and economic benefits have long been described $[2,3]$. It continues to be neglected and very often its public health impact is minimized by other priority infectious diseases like dengue, malaria, tuberculosis, and HIV. Reliable data indicating the actual incidence of human rabies and rabies risk exposures are often lacking or non-existent in many countries, leading to the global number of human deaths that is significantly underreported [3-5]. Canine rabies is not only a major burden in endemic countries where thousands of human deaths occur annually, but also in previously rabies-free areas where risks of re-emergence have been increasing over the last decade [6-10].

The burden of canine rabies is substantial, even though the disease is entirely preventable. Dealing effectively with the problem is contingent on investing in the control at the animal source, which has long been lacking. Long-term mass dog vaccination with high enough coverage could reduce health sector and societal costs with more rational and judicious use of postexposure vaccination $[11,12]$. Disease elimination is feasible with currently available vaccines and disease control methods; however, innovative financing models are required to overcome institutional barriers.

In 2001, the First WHO Interregional Consultation on Strategies for the Control and Elimination of Rabies in Asia laid down the impetus for many Asian countries to promote and pursue the elimination of canine rabies to eventually eliminate the disease in human populations [13]. Asian countries were urged to develop comprehensive national plans with improved access to modern human vaccines and application of new economical postexposure treatments, better disease diagnosis and surveillance, and processing of data at the national, regional, and global levels, intersectoral collaborative efforts for dog rabies control and plans to expand public and health care worker awareness regarding rabies control and prevention.

The 2008 Association of Southeast Asian Nations ASEAN Call for Action toward the elimination of rabies in the ASEAN Member states and the plus three countries (China, Japan, and Korea) by 2020 demonstrated the key importance attached to rabies control at a political level [14]. The ASEAN Rabies Elimination 
Strategy (ARES) was developed in 2015 to provide a strategic framework for the reduction and ultimate eradication of canine rabies in ASEAN Member States. The strategy describes an integrated One Health approach that brings together the necessary sociocultural, technical, organizational, and political pillars to address this challenge. The ARES was designed to complement the existing subregional frameworks developed to control and eliminate human rabies, such as those developed by the ASEAN Expert Group on Communicable Diseases (AEGCD) in 2010. In South Asia, considering the importance of consolidating achievements in rabies control in Member countries, the WHO Regional Office for Southeast Asia has developed a regional strategy for elimination of human rabies transmitted by dogs (2012) [15].

In the Middle East and Central Asia, human cases still occur, and dogs are the main vector. These regions plus countries of North Africa and Europe, belong to the Middle East and Eastern Europe Rabies Expert Bureau (MEEREB), an interregional rabies prevention and control network. In 2015, MEEREB has called for elimination of dog-transmitted rabies through vaccine and rabies immunoglobulin stockpiles and implementation of a One Health approach to achieve rabies eradication [16].

\section{Animal Rabies Prevention}

Across the continent, there is a marked increase in community-based initiatives for domestic animal vaccination and control with increased government support to funding and better program implementation. Animal rabies control activities vary across the region [17-20]. Many national and subnational programs and demonstration projects have proven that proactive mass dog vaccination is much more effective at controlling rabies and less costly than campaigns that vaccinate in response to the occurrence of cases. Control through proactive vaccination followed by two years of continuous monitoring and vaccination should be sufficient to guarantee elimination from any area not subject to repeat introductions [6]. The degree of success of national and global canine rabies elimination efforts, however, depend heavily on effective epidemiological surveillance, which should ensure that intervention impacts can be monitored through time and outbreak responses initiated where necessary. It is recommended that rabies control programs ought to be able to maintain surveillance levels that detect at least 5\% (and ideally 10\%) of all cases to improve their prospects of eliminating rabies, and this can be achieved through greater intersectoral collaboration [6].

Rabies is a community-based problem that requires a well-organized and funded community-based approach. Many countries need to strengthen their communitybased programs and implementation platforms, especially where government lacks the capacity and effective governance to mobilize community efforts $[18,19]$. In these settings, well-organized community efforts that aim to support or augment existing government rabies elimination programs are much desired. In countries such as Indonesia, Philippines, Sri Lanka, and Thailand, these are often facilitated by 
nongovernment organizations and civil society organizations closely coordinating with the local government and private sector groups.

There are practical bottom-up approaches that can be appreciated by governments. Effective community-based approach seeks to strengthen the capacity of families, individuals, organizations, institutions, and systems to support disease programs and outbreak responses. It is expected to contribute in programming to reduce rabies risk and address community vulnerabilities, and enhance community and institutional resilience, being sensitive to the issues directly confronting communities, and desiring to support families and all sectors involved (whole-ofsociety) to take the necessary actions to reduce dog bites and rabies transmission risks. Working through the lowest unit of a community, the family or household unit, is central, as "more resilient families are the foundation of more resilient communities." Individual family units should be fully aware of rabies threats and the required interventions and be the first to take action when these threats appear, such as reporting dog bite incidents to community leaders. The ability of a nation to eliminate rabies starts at household levels-with family members understanding the risks of rabies and being able to systematically mitigate spread, and therefore ensure neighborhood rabies security. Fostering private sector commitment to building and empowering communities through their corporate and human resource is another key element to effective community efforts.

An integrated approach is the most effective way of protecting humans from canine rabies, as the infection is maintained in domestic dog populations [11]. A number of countries have achieved considerable success in canine rabies elimination through mass dog vaccination. The feasibility and cost effectiveness of this approach have been strongly advocated in recent years, with major international public and animal health organizations declaring global canine rabies elimination as a realistic goal.

\section{Human Rabies Prevention}

\section{Postexposure Prophylaxis}

Significant progress in the production of rabies vaccines for human use that are low cost, rapidly immunogenic, safe and practical to use has led to increasing accessibility to timely and appropriate PEP. In the early 1990s, Thailand pioneered the PEP intradermal (ID) regimens using cell culture-derived vaccines [21]. From the time it was endorsed by WHO in 1992, accessibility further improved due to increase in numbers of animal bite management centers, and better quality of services $[21,22]$. This eventually eased out the production of mammalian nerve tissuederived vaccines. In the Philippines and Sri Lanka, since mid-1990s, animal bite treatment centers were established in government hospitals and major health facilities [21, 22]. Minimum essentials include training for MDs and nurses in the proper management of patients and rabies exposures, cold chain, and systematic 
recordkeeping or registry. To date, ID regimens are the first line in majority of the national rabies prophylaxis protocols and recommendations.

Rabies immunoglobulin of equine origin (ERIG) are in short supply throughout the world and particularly in Asia, the demand is high [19]. ERIG available in Asia is either manufactured in Europe, India, or China. Though ERIG is considerably cheaper than human origin immune globulin, modern production of immune sera generates highly purified and safer products of better quality. Producers of ERIG should be encouraged to continuously aim for consistent antibody levels and the least incidence of adverse reactions among patients.

\section{Pre-exposure Prophylaxis}

Current recommendations for pre-exposure schedule use ID injections of cell culture vaccines as a cost-reducing alternative for developing countries. As a strategy to augment human rabies prevention measures, childhood rabies immunization has been included in the national programs of countries like the Philippines and Vietnam.

\section{Human Rabies Vaccine Production in Asia}

Human vaccine production capacities of Asian countries have improved greatly. Rabies vaccine supplies come from a mix of private and public manufacturers in several Asian counties and are mainly for domestic use, but some manufacturers have the potential to export vaccines. Over the last decade, both private and public vaccine manufacturers in Asia have exerted extra efforts to meet stricter government registration requirements as countries adhere to international and regional GMP (Good Manufacturing Practice) standards and vie for WHO pre-qualification. The National Regulatory Authority (NRA) in most countries generally enforces their local GMP standards, which tend to be stricter and more demanding to the producers [23]. The procedure for approval of newly introduced vaccines is very much in place, involving the conduct of complete preclinical and clinical testing and establishing lot consistency prior to approval for marketing. Most countries have functional NRAs/National Control Laboratories (NCLs), which provide overall control on the vaccine production process and final product quality. Exceptionally, a few countries still lack the capacity to perform rigid laboratory testing.

Demand and Supply Human rabies vaccine manufacturers are growing in number most markedly in China and India with more than 15 manufacturers serving a combined population of more than 2 billion with an estimated demand for rabies vaccines of about 30 million doses or about 6 million full-course treatments per year. The vaccine production levels typically range from 100,000 doses to 10 million doses per year (for cell-based production facilities), with some producers in China and India upgrading their capacities to produce more [23]. 
Vaccine Types China and India discontinued the production of mammalian nerve tissue origin (NTO) vaccines in 2001, and Vietnam stopped producing the sucklingmouse brain vaccine, which has been in use over 30 years, in 2005 [23]. It now imports cell line-based vaccines, and they have also modernized their main vaccine manufacturing plant (GMP compliant) located in Hanoi. All these manufacturers follow the WHO standard requirements, as well as refer to USP requirements. There are essentially 5 types of modern vaccines, according to the substrate, that are produced mainly in China and India, namely the Primary Hamster Kidney (PHK) cell, Vero cell, Human diploid cell (MRC-5), Embryonated duck egg, and Chick embryo cell. Of these, the Vero cell is the only continuous (animal) cell line. All these vaccine types undergo concentration and purification processes by either zonal centrifugation or tangential filtration and gel chromatography. The virus strains being used include the PV (Pasteur Institute Paris or CDC) and PM (Wistar Institute), and in China they also use their locally derived strains. Vaccine preparations are either liquid or freeze-dried single dose $(0.5$ or $1.0 \mathrm{ml})$ in glass vials and are administered according to the Essen regimen [23].

Quality Asian vaccine manufacturers generally apply in-process control measures that include sterility tests, ELISA, SRD, and NIH potency testing. National Regulatory Authorities only issue marketing licenses if complete testing of vaccines, including preclinical and clinical studies, has been conducted. Laboratory testing regimens applied essentially follow WHO requirements, and Lot Release systems are being constantly reviewed and modified. In China, a system of random testing of production lots and post-market surveillance/testing and product recall are being strictly put in place. Random lot testing generally includes tests for sterility, safety, and potency (NIH method). Most rabies vaccine manufacturers (public and private) seek to be WHO pre-qualified as they consider it advantageous to the marketing and worldwide distribution of their products [23].

Costs In China and India, the cost of modern locally produced vaccines range from 3 to 7 USD per dose. In China, imported vaccines are 10-14 USD per dose. In Southeast Asia, imported vaccines typically range from 3 to 10 USD per dose.

Hold Backs and the Way Forward Some countries that intend to start their local modern rabies vaccine production need support to establish cell line-based vaccine production, such as seed virus, cells, technology transfer, and funding for equipment or facilities. As WHO prequalification is sought for rabies vaccines, manufacturers observe that the process takes too long. As more new manufacturers join in, the demand for training of personnel on GMP must be addressed by all stakeholders. The reliability of the currently prescribed NIH potency test is a major problem that manufacturers face in the production and control of rabies vaccines. Essentially the $\mathrm{NIH}$ test gives varying results depending on the laboratory and the status of the mice which the test utilizes. Some manufacturers have also questioned the need to conduct the stability testing of vaccines on a per batch basis [23]. 
In general, countries are able to follow the WHO requirements for human rabies vaccine production. The complete replacement of NTO vaccine with cell line-based rabies vaccines has been accelerated, as India and China have demonstrated the feasibility of domestic commercial vaccine production. Countries generally have the desire to produce better quality vaccines but are concerned about the effect on supply, and how to get the production of cell line-based vaccines started. Regional supplies of relatively inexpensive vaccines will surely influence the decisions of countries to produce their own cell line-based vaccines. It would be advantageous to countries if WHO introduced a system of recognizing (qualifying) domestically produced vaccines using various types of cell substrate, and encourage exports where appropriate, principally to lower the world price of human rabies vaccines.

\section{Advocacy and Public Information and Education}

To increase awareness and enhance community participation and support, public information and education are necessary. Components of the information campaign generally have discussions on rabies as a fatal disease, its epidemiology, and its prevention and control, the disease control program in general and related national and local rabies ordinances as they support the program implementation and responsible pet ownership. With the realization of the impact of rabies in daily lives, and that pets can be a source of human infection, implementing community and schoolbased programs were relatively easy to roll out. Volunteerism, active engagement, and willingness to pay of people in the program stems likewise from communitybased initiatives [24].

Community-based programs found all over Asia concentrate on campaigns using multimedia (television, radio, newspapers, Internet/mobile devices), display of posters and banners in strategic areas, distribution of flyers and other materials, public hearings of local ordinances and hosting of village assemblies. Some educational campaigns are often conducted at various government offices and in churches or other religious structures. Generally, celebrations like the World Rabies Day are observed to remind people of the continual threat of rabies and the importance of the program to control and eliminate the disease. School-based rabies educational programs, designed to improve awareness about rabies prevention among children are common in countries like India, Philippines, Thailand, and Vietnam. These were mostly developed and are implemented with the Ministry of Education and in coordination with the Ministries of Health and Agriculture. In the Philippines, the integration of rabies education into the school curriculum was initially developed by the Department of Health's National Rabies Control Program in 2006 [25]. Lesson plans prepared by school teachers integrated facts and figures about rabies, and lessons on responsible pet ownership. Activities for the children involve fun educational events to celebrate the bond between children and pets. The power of the youth must be harnessed. Lessons taught in interactive school programs could be brought into households and be ingrained in family values. Rabies awareness in youth and adolescents will ripple through the entire family unit, thereby ensuring 
sustainable rabies interventions in future generations. The youth can proactively be involved in dog vaccination and control.

\section{Governance, Policy, and Funding}

Governments that are committed to implementing disease control programs provide the institutional framework, legislation and policies, infrastructure and logistics, human resources, and budget appropriation. The legal framework for implementing rabies prevention and control programs is already in place in most countries in Asia. National legislation defines the roles and responsibilities of the councils including dog and dog owner registration; collection of registration fees; animal population control; dog vaccination; surveillance of human and animal rabies and exposures; settlement of disputes/agreements between bite victims and dog owners; and promotion of responsible dog ownership. Funds for disease control programs are traditionally sourced from local and national governments, and international development aid. Actual implementation of intersectoral rabies control programs often requires and depends on regular budget allocation as mandated by law. International aid agencies and nonprofit organizations offer funding and technical inputs, pooling of resources, set guidelines, and standards, have monitoring and evaluation mechanisms and often act as an intermediary between donors and government.

\section{Whole-of-Society Stakeholder Involvement}

In any disease control program, wide stakeholders' involvement is critical [26]. It is important to bring together key stakeholders from business and the public sector to discuss health security and the importance of establishing public-private partnerships. Contributions from private organizations, including businesses, academe, and civil society, can be tangible and intangible. Tangible efforts are generally in the form of donations in kind or money. Intangibles such as voluntary efforts should be maximized. The intense involvement of the local communities has served as a conduit for business sectors, nongovernment organizations, academic institutions, and civil society organizations to extend their financial and technical assistance to the government. The national government agencies can sustain the standardized approaches to rabies control and elimination and promote how to start the public-private partnership that would ensure sustained intervention. Such technical and administrative conduits are essential and beneficial to all stakeholders, providing the credibility and quality assurance that is directly rooted in the day-today field operations.

There are numerous examples of public-private partnerships that contribute to public program implementations, support research and promote policy development in Bali, Indonesia, India, Sri Lanka, Philippines, Thailand, and Vietnam [19]. A number of rabies control programs in humans and animals have sourced funds from different sectors at different levels. The range of sources could be from the grassroots 
to the corporate and people's organizations. General support to local governments given by partner organizations includes community mobilization, volunteer services, and materials donations. The business sector gives direct donations or embarks in joint ventures. The academe conducts research and offers technical inputs, voluntary services, and student manpower. The community contributes taxes, fees for service, donations, and volunteer manpower.

Field implementers and partner communities often face constraints such as high operational cost, wide regions of coverage and labor intensity. Many innovative approaches have been attempted to overcome these problems. There are numerous lessons of good practices learnt from experience. An example of a successful, sustainable community-based integrated rabies control program is the Bohol Rabies elimination program, implemented as a partnership between the provincial government, the national government line agencies (Health, Agriculture, Education, Interior, and Local Government) and a few nonprofit organizations. The project brought together educators, physicians, veterinarians, government officials, community leaders and the general public, and aligned them for coordinated effort [25]. This program produced a significant shift in rabies control, from government-dependent implementation to a community-led movement. Collateral benefits included better conditions for animal welfare, more responsible pet ownership, and improved public safety. Ownership of the program at the community level has assured more engaged field operations and sustainability. Attaining the goal of rabies control and eventual freedom from disease became a shared concern.

There are challenges though to public-private partnerships. The continued assurance of private-sourced funds depends on the effort to acquire these; thus, fund sourcing must be a full-time effort that requires a wide range of committed stakeholders. The credibility that has been established through successful local programs facilitates fund sourcing. Field experience showed that there could be disincentives to provision of external assistance including an uncertain political environment, lack of political support, and inadequate counterpart funds [7, 9].

The key to the success of a public-private partnership model is the strategic partnership among the community-based stakeholders with sound technical and operational capabilities to implement the rabies control and elimination program framework and strategic plan. The partnership ensures evidence-based and informed program planning, institutionalized organization, policies, and implementation mechanisms, the setting in place of clear performance indicators, and uninterrupted resource inputs. The key steps in project integration within the local system is the identification of key persons or technical and political champions, clear and functional feedback channels among partners (e.g., internal and external monitoring), and encouraging government empowerment and program ownership, stakeholder participation and formally defining roles and responsibilities of stakeholders through memoranda of understanding. Increased public awareness and understanding enhance willingness to pay and contribute for public good. 


\section{Rabies and the One Health Approach in Asia}

Program sustainability is a critically important issue for all public health programs, but especially for resource-poor countries with limited budgets and many problems to resolve. Thus, a successful rabies prevention and control program must be built around integration and the strengthening of intersectoral and transdisciplinary collaboration and cooperation between several societal components [11, 26, 27].

The ASEAN Rabies Elimination Strategy gives particular importance to the organizational and One Health framework for rabies elimination [14]. As an example to understand better, the expansiveness of One Health challenges: In dealing with urban rabies threats, it is recognized that the best single approach is to attack the disease at its source, that is, to eliminate dog-mediated rabies. Eliminating dog rabies greatly reduces the need for postexposure human prophylaxis, at least at some point in time if the process is executed systematically. In this regard, the health sector has been at the forefront of rabies elimination programs. While this traditional principle of rabies elimination is proven to be one of the most well-based and sound of disease control strategies, in reality program implementations are confounded with complexities, resulting in more failures than successes (with only a few established and emerging exemptions). The failures have often been associated with the re-emergence of rabies after it had been temporarily eliminated in the dog population. Even areas (e.g., islands) once rabies-free have encountered emergence and endemic spread of urban rabies [8-10]. This has been the general situation for many decades. While the prescribed solution is sound and tested, i.e., elimination of rabies at-source, in the overall process, whole-of-society must deal with the complexities of prevailing urban rabies. Detailed scientific argument is not necessary to point out that poverty is a strong driver of rabies endemicity. For example, the massive proliferation of slum areas is directly proportional to rabies proliferation. The survival priorities of people dictate their health and wellbeing-seeking behaviors; obviously, food and shelter come first to those who are hungry and cold. In the same way, hungry stray dogs seek food and shelter, and the proliferations of garbage and market wastes drive these behaviors. Populations, whose general health and wellbeing are deteriorating, will be further drawn into the state of poverty. Where there are people who (must) eat dogs, there will be those who propagate and market dogs legally or illegally. There are a number of undesirable reasons why dogs are able to cross boundaries and islands. And there will always be bad governance that reciprocate bad community participation/cooperation. Such complexities are too numerous to mention all here but are at the heart of why programs fail. Very similar arguments also apply to the continued proliferation and emergence of other infectious diseases [28]. Most significantly, poverty dynamics clearly drive vulnerabilities to diseases [28-30], and these include (1) lack of adequate safe food and water; (2) lack of protection from harm such as exposure to pests, inclement weather, pollution, violence, stress, and disasters; (3) extreme social marginalization and deprivation of opportunities to earn a living, to be educated, to receive healthcare; and (4) infliction of collateral harm, especially to woman and children, the disabled and the elderly. Clearly, the determinants of infectious diseases are 
multifaceted and increasingly complex [31]. Poverty reduction is central, as generally, Poverty Alleviation means Vulnerability Reduction. This has been documented in relation to the likelihood of infectious diseases emergence in impoverished community settings [32].

Good governance, involving the highest inter-ministerial central body for One Health coordination backed up by legislation and a clear mandate, budget appropriation, resources mobilization, and pilots or model programs that lead to policy development, provide optimism to implementing comprehensive operational plans that are vertical and horizontal, national and sub-national. These are important institutional drivers and enablers for a sustainable public-private partnership.

Comprehensive rabies control programs should consider combining human, financial, and material resources with other interdisciplinary disease programs to benefit from synergy and maximization of shared resources. With the guidance of OIE, FAO, and WHO, governments, donors, foundations, and other private partners should be mobilized to sustain investment in canine rabies control and eventual elimination.

Pursuing the regional goal of rabies elimination cannot be taken lightly. Sustained investment mechanism and integrative efforts must be enabled, for instance, by the designation of a specifically mandated body, e.g., a Rabies or One Health Authority directly under the Office of the President or Prime Minister. Such body could be assigned a czar (secretary or minister level) and a dedicated budget for office and resources. It should be solely focused on rabies elimination (in the meantime), and collaborate as necessary with the health, veterinary, education, environment, industry, and other sectors on clearly defined parameters and terms, with its authority maintained at all levels, i.e., national to local. The structure and mechanism for this could be legislated. Such legislation, together with the creation of the One Health authority, will remain relevant to the continuous prevention, control, and eradication of any zoonoses threat (e.g., Ebola, influenza, SARS, MERS-CoV, malaria, leptospirosis) that potentially are pandemic threats. It is important to recognize the main justification for these radical recommendations which is: any country with a prevailing human rabies threat in this modern and highly connected world is considered a hindrance to global progress.

All stakeholders are specifically drawn to the enhancement of governance. This is to ensure a sustainable approach to comprehensive capacity strengthening and broader risk reduction in the context of community resilience and regional security. The overriding objective is to advocate for continued and better targeted funding to strengthen capacities to immediately and effectively detect, prevent, and prepare for and respond to any infectious disease/zoonosis outbreaks and similar major threats. Targeted initiatives must promote broad resilience objectives, cognizant that absolute efficiency of systems, especially in relation to widespread threats, is contingent on the interdependencies of sectoral and systems approaches, and the capacity to enable strategic systems synergies.

Whole-of-government/whole-of-society coordination, involving multi-sectors within communities, is key. Therefore, rabies and zoonoses preparedness needs to be integrated into emergency and crisis response systems. The systematic involvement of even the military should be pursued. 


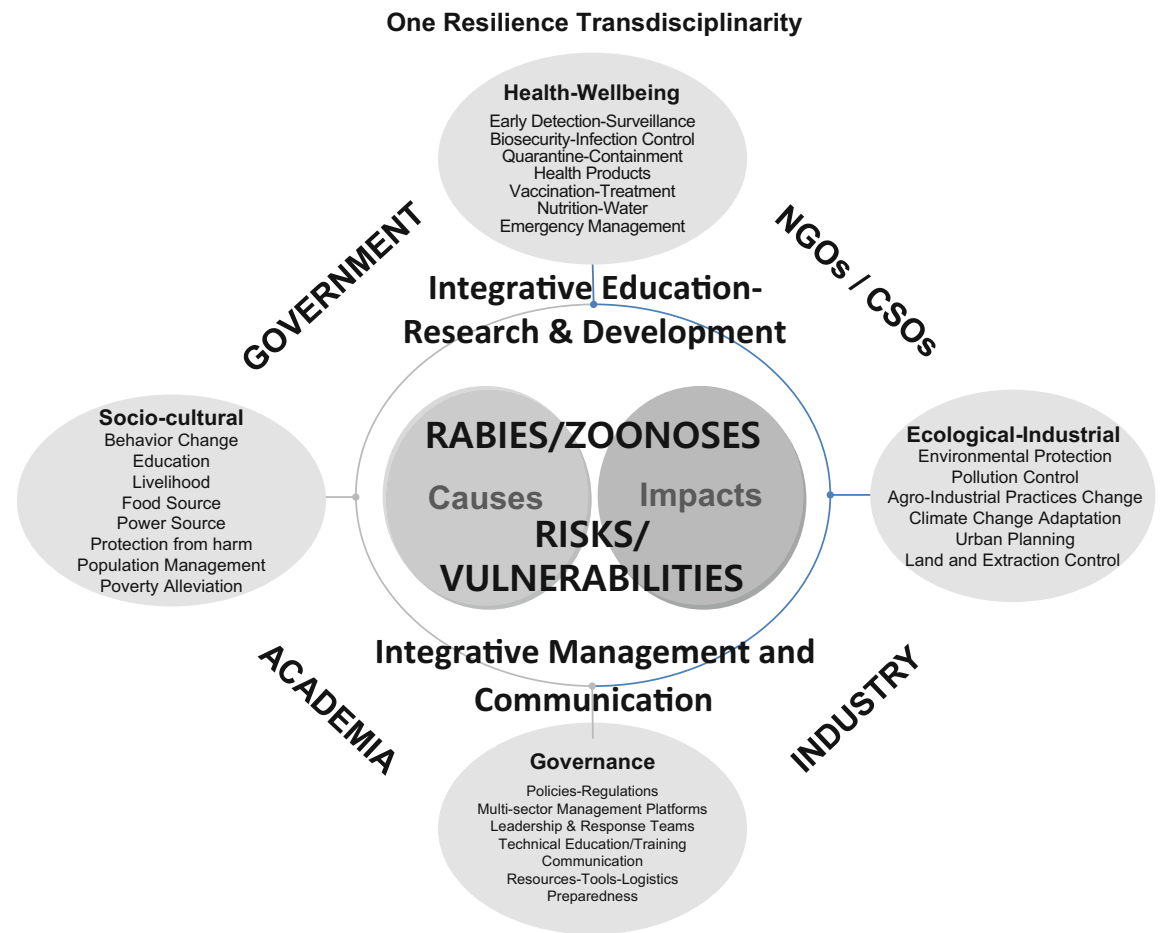

Fig. 1 Interactions among national and regional entities involved in the prevention and control of rabies and other zoonoses

The One Health Authority's core structure, functions, and capacities to plan, prepare, mitigate risk, and respond to threats through its dedicated rapid response teams should be sustainable. These must integrate into the broader whole-of-society platform and proposed "One Resilience" approach [33] to effective interactions among national and regional entities involved in the prevention and control of rabies and other zoonoses (depicted in Fig. 1), to the extent that all actors understand their roles and are enabled to effectively respond when major threats strike, so that normal operations, economic activities, and livelihood are protected and sustained.

\section{References}

1. Hampson K, Coudeville L, Lembo T, Sambo M, Kie er A, Attlan M, et al. Estimating the global burden of endemic canine rabies. PLoS Negl Trop Dis. 2015;9(4):e0003709. https://doi.org/10. 1371/journal.pntd.0003709.

2. Fishbein DB, Miranda NJ, Merrill P, Camba RA, Meltzer M, et al. Rabies control in the Republic of Philippines: benefits and costs of elimination. Vaccine. 1991;9:581-7. 
3. Knobel DL, Cleaveland S, Coleman PG, Fèvre EM, Meltzer MI, Miranda MEG, et al. Re-evaluating the burden of rabies in Africa and Asia. Bull World Health Organ. 2005;83 (5):360-8.

4. Tenzin WMP. Review of rabies epidemiology and control in South, South East and East Asia: past, present and prospects for elimination. Zoonoses Public Health. 2012;59:451-67.

5. Dodet B, Fooks AR, Miller T, Tordo N, editors. Towards the elimination of rabies in Eurasia. Basel: Karger; 2008. p. 95-121.

6. Townsend SE, Lembo T, Cleaveland S, Meslin FX, Miranda ME, Putra AAG, Haydon DT, Hampson K. Surveillance guidelines for disease elimination: a case study of canine rabies. Comp Immunol Microbiol Infect Dis. 2013;36(3):249-61.

7. Windiyaningsih C, Wilde H, Meslin FX, Suroso T, Widarso HS. The rabies epidemic on Flores Island, Indonesia (1998-2003). J Med Assoc Thai. 2004;87:1389-93.

8. Tenzin DNK, Dorjee J, Ward MP. Re-emergence of rabies in dogs and other domestic animals in eastern Bhutan, 2005-2007. Epidemiol Infect. 2011;139:220-5.

9. Susilawathi NM, Darwinata AE, Dwija IBNP, Budayanti NS, Wirasandhi GAK, Subrata K, et al. Epidemiological and clinical features of human rabies cases in Bali 2008-2010. BMC Infect Dis. 2012;12:81. https://doi.org/10.1186/1471-2334-12-81.

10. Liu C-H. History of rabies control in Taiwan and China. Taiwan Epidemiol Bull. 2013;29: S44-52.

11. Léchenne M, Miranda ME, Zinsstag J. Integrated rabies control. In: Zinsstag J, Schelling E, Waltner-Toews D, Whittaker M, Tanner M, editors. One Health: the theory and practice of integrated health approaches. Wallingford: CABI; 2015. p. 176-89.

12. Miranda L, Miranda M, Hatch B, Deray R, Shwiff S, Roces M, et al. Towards canine rabies elimination in Cebu, Philippines: assessment of health economic data. Transbound Emerg Dis. 2015;64(1):121-9. https://doi.org/10.1111/tbed.12350.

13. WHO/CDS/CSR/EPH/2002.8. Strategies for the control and elimination of rabies in Asia. Report of a WHO Interregional Consultation. Geneva, Switzerland, 17-21 Jul 2001. Geneva: WHO; 2001.

14. OIE World Organisation for Animal Health, OIE Sub-Regional Representation for South-East Asia (OIE SRR-SEA). 2016. ASEAN rabies elimination strategy. Jakarta: ASEAN Secretariat; Dec 2016.

15. WHO Regional Office for Southeast Asia. Strategic framework for elimination of human rabies transmitted by dogs in the South-East Asia region. New Delhi: WHO; 2012.

16. Picot V, Rasuli A, Abella-Rider A, Saadatian-Elahi M, et al. MEEREB report of the Third Meeting of the Middle East and Eastern Europe rabies expert bureau, Lyon, France (7-8 April 2015). J Infect Public Health. 2015;10(6):695-701. https://doi.org/10.1016/j.jiph.2017.03.005

17. Hu RL, Fooks AR, Zhang SF, Liu Y, Zhang F. Inferior rabies vaccine quality and low immunization coverage in dogs (Canis familiaris) in China. Epidemiol Infect. 2008;136 (11):1556-63.

18. WHO. WHO expert consultation on rabies. WHO Technical Report Series no. 931. Geneva: WHO; 1997.

19. WHO. WHO Expert Consultation on rabies: second report. WHO Technical Report Series no. 982. Geneva: WHO; 2013.

20. OIE/WHO/EU. Towards the elimination of rabies in Eurasia: joint OIE/WHO/EU International Conference, Paris; May 2007: Proceedings. Developments in biologicals, Vol. 131.

21. WHO. Report of a WHO Consultation on Intradermal Applications of Human Rabies Vaccines, Geneva, 13-14 March 1995. Geneva: WHO; 1995.

22. WHO. Rabies vaccines: WHO position paper. Weekly Epidemiological Record 85:309-20; 2010.

23. WHO. WHO Expert Committee on Biological Standardization. Fifty sixth Report. WHO Technical Report Series no. 941. Geneva: WHO; 2005. 
24. Birhane MG, Miranda MEG, Dyer JL, Blanton JD, Recuenco S. Willingness to pay for dog rabies vaccine and registration in Ilocos Norte, Philippines (2012). PLoS Negl Trop Dis. 2016;10(3):e0004486. https://doi.org/10.1371/journal.pntd.0004486.

25. Lapiz SMD, Miranda MEG, Garcia RG, Daguro LI, Paman MD, Madrinan FP, et al. Implementation of an intersectoral program to eliminate human and canine rabies: the Bohol Rabies Prevention and Elimination Project. PLoS Negl Trop Dis. 2012;6:e1891. https://doi.org/10. 1371/journal.pntd.0001891.

26. Lembo T, Attlan M, Bourhy H, et al. Renewed global partnerships and redesigned roadmaps for rabies prevention and control. Vet Med Int. 2011;2011:923149. https://doi.org/10.4061/2011/ 923149.

27. Miranda NLJ. Integrative societal resilience or 'one resilience' approach: towards optimal health and wellbeing. Int J Philip Sci Technol. 2015;8(2):44-7.. http://philscitech.org/2015/1/ 2/023.html

28. Farmer P. Infections and inequalities: the modern plagues. Berkeley: University of California Press; 1999.

29. Miranda NLJ. Sustained global attention to emerging pandemic threats and risks: the need to strengthen one health systems and whole-of-society preparedness. Int J Philip Sci Technol. 2015;8(1):58-9. http://philscitech.org/2015/1/1/013.html

30. WHO. Global report for research on infectious diseases of poverty. Special programme for research and training in tropical diseases; 2012.

31. Ghose B, Seydou I, Sharmistha G. Social determinants of infectious diseases in South Asia. Int Schol Res Notice. 2014;2014:135243. https://doi.org/10.1155/2014/135243.

32. Grace D, Mutua F, Ochungo P Mapping of poverty and likely zoonoses hotspots. DFID Zoonoses Report, 4:1-119; 2012.

33. Miranda NLJ. Biosecurity within one resilience. Paper presented at the ASEAN Regional Forum (ARF) cross-sectoral security cooperation on bio-preparedness and disaster response workshop, Manila, Philippines; August 2014. 